\title{
Engineering Human Tissues
}

\section{Gordana Vunjak- Novaković}

Professor, Department of Biomedical

Engineering

Director, Laboratory for Stem Cells

and Tissue Engineering

Columbia University

622 west $168^{\text {th }}$ Street, VC12-234

New York NY 10032

gv2131@columbia.edu

www.bme.columbia.edu/gvnweb

\begin{abstract}
There is no tissue repair without cells, either recruited from the host or supplied from an external source. It is now feasible to induce tissue development and remodeling in certain cases by application of cells and matrix along with molecular and physical regulatory factors that direct native development. Concepts intrinsic to developmental (and adult) biology are thus crucial for developing the new generation of tissue engineering technologies, by temporal and spatial signaling, niche development, and an integrated use of regulatory factors. This interdisciplinary research, which lies at the interfaces between biology, bioengineering and clinical sciences, is essential for driving the field forward.
\end{abstract}

(Scr Med 2010;41:83-7)
Accepted for publication

August 29, 2010
Clinical need. Over the last two decades, regenerative medicine has changed dramatically, largely due to tissue engineering, a new interdisciplinary area. In our aging population with increasing expectations for a high quality of life, there is an increasing need to repair tissues lost to congenital abnormalities, injury, or disease. Engineered tissues also find roles as controllable models for fundamental biological research, study of disease, and testing of drugs.

Some living tissues can be replaced by a prosthetic device, such as an artificial hip or knee. Transplantation of living tissues and organs is generally considered only after all other options are exhausted. It can be life saving, but there are certain serious limitations. For example, autologous transplants are limited to only a few types of tissues (bone, skin); in addition, only relatively small grafts can be harvested, and the required surgical shaping of the harvested tissue as well as donor side morbidity and long recovery times may limit effective transplantation. In contrast, allogeneic transplantations (the only option for heart or lung transplantation) are limited by the availability of matching organs, and the need for long-term immunosuppresion. The ability to make functional human tissues - ideally, from the patient's own cells-with shape and properties corresponding exactly to the defect to be repaired would provide biological "spare parts" with the potential for reconstructing the original tissue. This is precisely the long-term goal of tissue engineering. While still in its infancy, with only a few successes and many challenges to be overcome, tissue engineering offers significant promise.
Tissue culture. It is not easy to say when the idea of replacing human tissues was born, as its roots can be traced all the way back to ancient times. From the Egyptian Sphinx and Greek Chimera to the modern recipients of transplanted organs, tissue exchange has inspired and intrigued people for centuries. There are records by native Indians of attempted nose transplants as early as $1000 \mathrm{BC}$, and people of ancient China tried heart repair surgery using homemade anesthetics.

The cell culture dishes we use today evolved from glass dishes made in the $19^{\text {th }}$ century by Julius Petri, assistant to the famous Robert Koch. Petri named the dishes after himself and used them for bacterial cultures. The first mammalian tissue culture was done in 1907 by Ross Harrison, ${ }^{1}$ who studied the outgrowth of peripheral nerves from embryonic tissues. This is still one of the most difficult in vitro culture systems. The field advanced through extensions of Harrison's techniques to the culture of adult mammalian tissues and the use of perfusion to keep these tissues alive. Charles Lindbergh, a cell biologist and aviator, who worked with cardiologist and Nobel Laureate, Alexis Carrell, contributed significantly to this field.

Identification of families of growth factors in the 1960s, followed by rapid advances in biology in 1970s, set the stage for integration of bioengineering, biological and clinical disciplines in the 1980 s.

The field of tissue engineering. The new discipline of tissue engineering was officially established at the Lake 


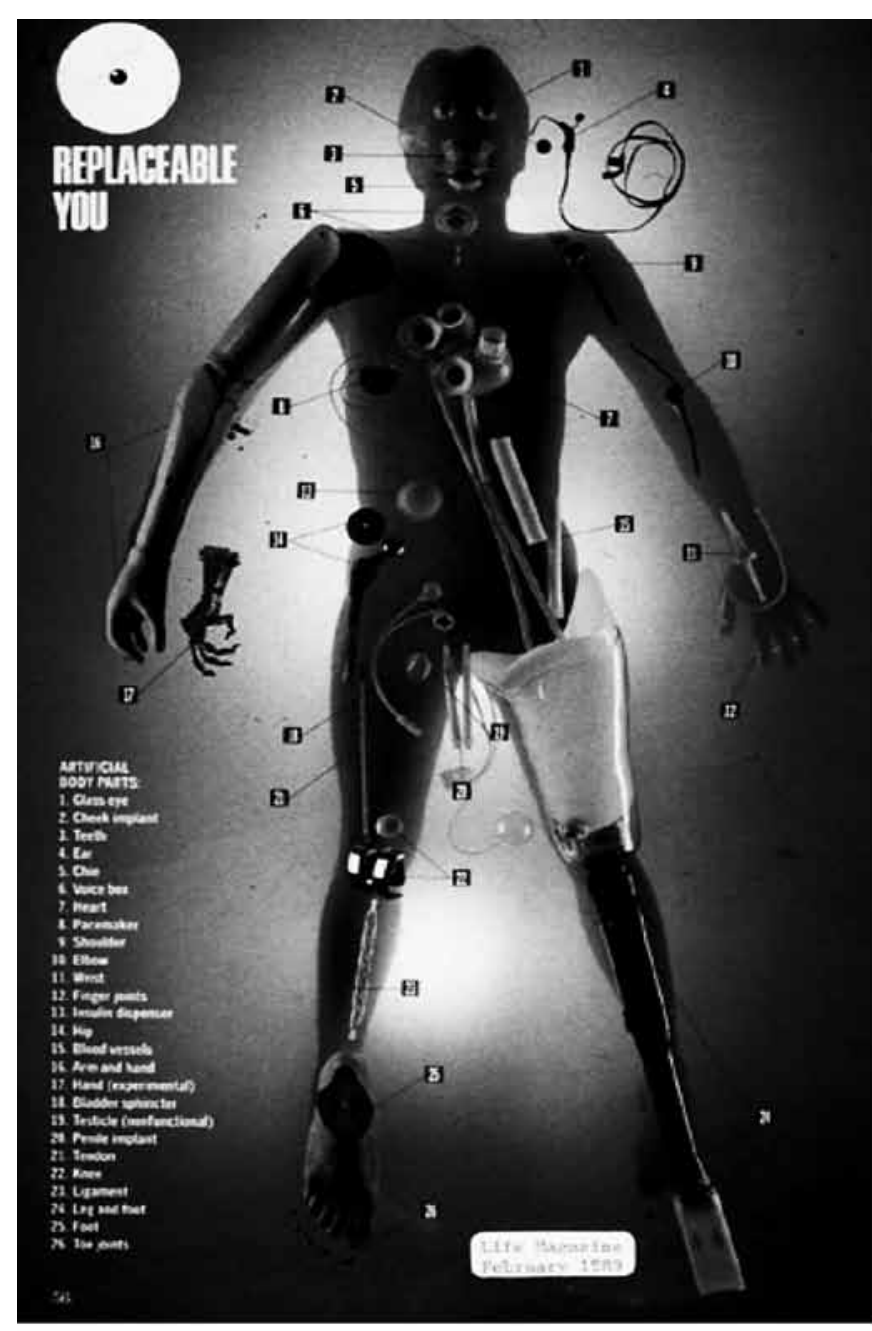

a

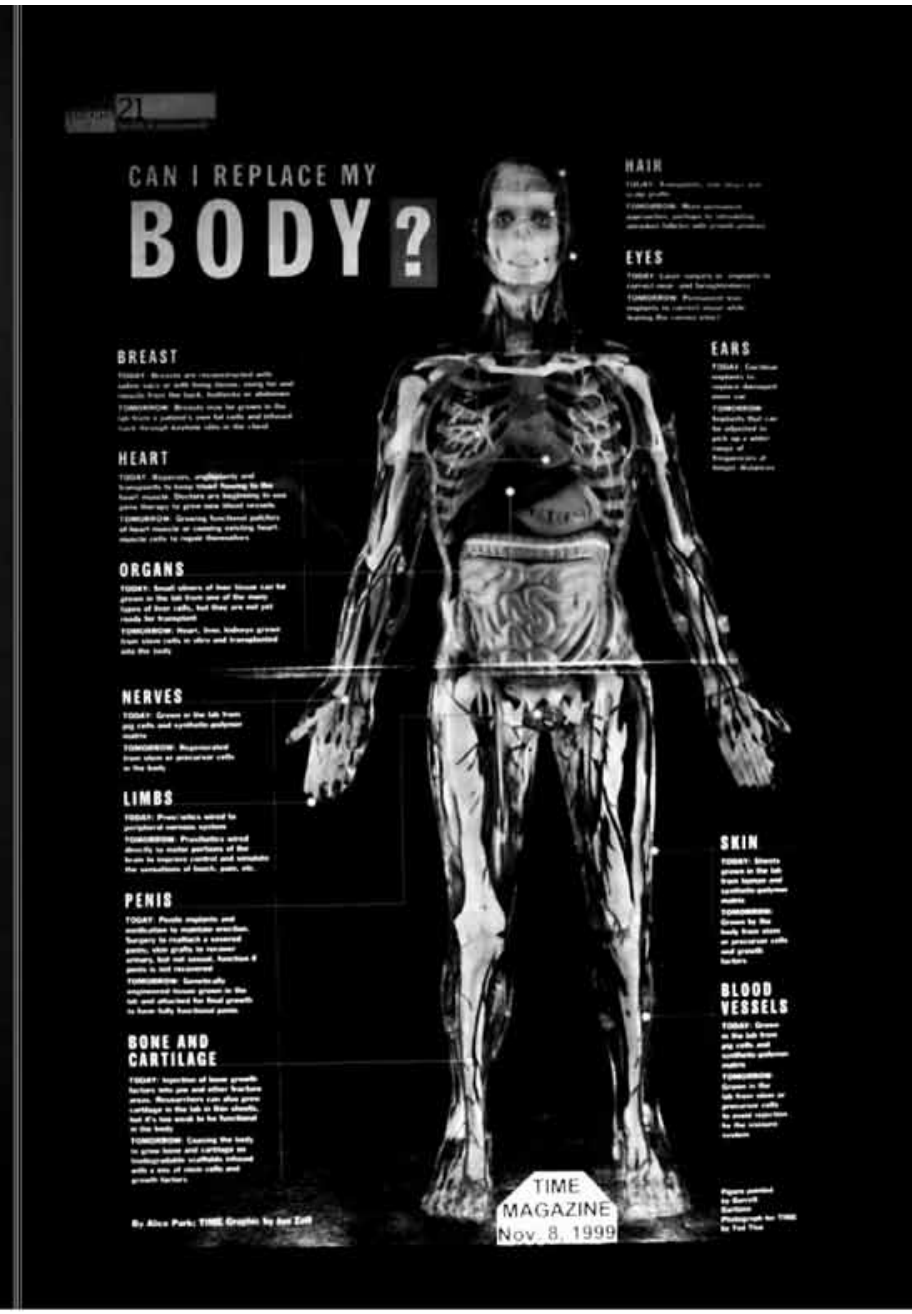

b

Figure 1. Tissue engineering: the beginning. (a) In 1989, Life magazine surveyed the available options for tissue replacement. A Robocop type of picture represents the concept accepted by the scientific community at that time. All tissue replacements were made of ceramics, metal and plastics. (b) Ten years later, a similar survey by Time magazine resulted in a "Bionic human," where all replacement tissues were fully biological. These two surveys, 10 years apart, illustrate the shift in the perception of regenerative medicine.

Tahoe conference in 1988: "Tissue Engineering is the application of principles and methods of engineering and life sciences toward fundamental understanding of structurefunction relationships in normal and pathological mammalian tissues and the development of biological substitutes to restore, maintain, or improve tissue function." This original definition still reflects the unifying concept of the tissue engineering.

Two articles from the popular press show how the mindset of the scientific community shifted between the 1980 s and 1990s. At the end of 1989, Life magazine ran a feature entitled "Replaceable You" that described existing options for replacing tissues and organs. Life's "robocop" type figure (Figure 1a) embodied the collective views to date: all tis- sue replacements were artificial and made of metal, ceramics and plastics. Exactly 10 years later, Time magazine ran a similar feature entitled "Can I replace my body?" The answer was strikingly different this time: all tissue replacements were biological (Figure 1b). Although the featured tissues were not necessarily available (even now, only a few grafts are available for clinical use) the focus changed in the direction of engineering human tissues "on-demand." The 1990s marked the start of a fast growing stream of publications - from only about a dozen in 1991 to many thousands in 2010-with the first review of the field appearing in $1993 .{ }^{2}$ As the field continues to evolve, it is fair to say that we are at the beginning of a new era. ${ }^{3-5}$ Today, tissue engineering has the potential to provide customdesigned tissues that can meet many needs, from acute 
surgical repair to routine "maintenance" of our tissue and organs for another lifetime of function.

The concept. In a developing organism, tissues emerge from coordinated sequences of cell renewal, differentiation and assembly that are orchestrated by cascades of multiple regulatory factors. The living cell is the only real "tissue engineer." We simply provide an instructive environment in which the cells can build tissue. It is important to understand that the cells respond to the entire context of their environment: surrounding cells, the structure and mechanics of an extracellular matrix, as well as to concentrations of oxygen, nutrients and growth factors and physical signals (flow-induced, mechanical and electrical). We can assume that the same factors determine cell function in vivo (during native tissue development and remodeling) and in vitro (in engineered tissues). These two premises are the basis for a "biomimetic," or biologically inspired, design of tissue engineering systems.
It has been argued that we need a new generation of culture systems, "something between a Petri dish and a mouse," to correspond to a cell's authentic environment in a living organism. ${ }^{6}$ In particular, in order to unlock the full potential of stem cells, we need to reconstruct certain aspects of their dynamic native environments that induce them to replicate, differentiate and assemble into tissues.

The overall concept of tissue engineering is remarkably simple: cells are cultured on a scaffold of biomaterial (a surrogate of native tissue matrix that provides a structure for cell attachment and tissue formation) in a bioreactor (controlled culture environment that provides nutrients and physical signals similar to the body environment) (Figure 2). Manipulation of the scaffold and bioreactor can "instruct" pluripotent cells to differentiate into the right cell types, in the right location within the scaffold and the right time, for a tissue structure to be assembled. However, each tissue has its own demands for cell types

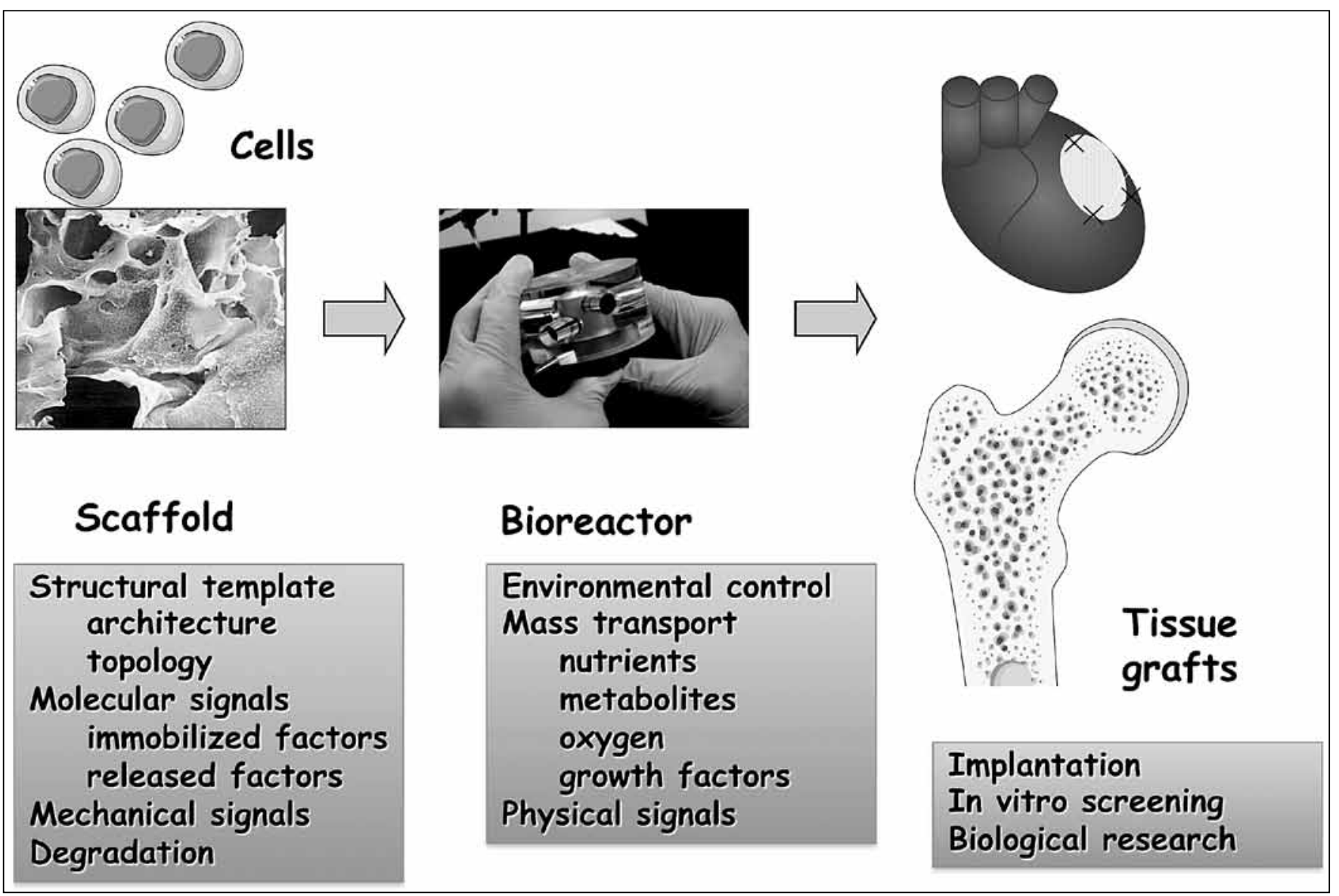

Figure 2. Tissue engineering paradigm. Living cells (either differentiated or progenitor/stem cells) are seeded onto a biomaterial scaffold and cultured in a bioreactor to carry out the process of tissue formation. The scaffold provides a template for cell attachment and tissue formation through its structural properties (overall architecture, surface properties), molecular factors (immobilized and released molecules and cell-receptor ligands), mechanical properties (stiffness and elasticity), and it degrades in parallel with deposition of new tissue matrix. The bioreactor provides the entire milieu of environmental conditions necessary for regulating cell differentiation and functional assembly. It controls the cell environment (temperature, $\mathrm{pH}, \mathrm{medium}$ composition) and application of physical stimuli (hydrodynamic shear, mechanical stretch, compression, electrical stimulation). 
and sources, scaffold properties and environmental conditions. All of these factors must be established in a bioreactor, as detailed below.

Cells. Cells are central to any of our efforts to grow tissues or to develop platforms for therapeutic screening, and the choice of cells will depend on the type of tissue that is to be engineered. For example, one can induce cells harvested from a patient's bone marrow ${ }^{7}$ or fat to form bone tissue in vitro. ${ }^{8}$ However, the capability of adult stem cells to form other tissues of interest, such as heart muscle, pancreatic beta cells, or neural tissue has not been established. Instead, stem cells from more immature sources must be used..$^{8,9}$ The so called iPS cells are particularly promising. These are cells derived by reprogramming of adult cells (usually skin fibroblasts) to become embryonic-like; in this state they are capable of differentiating into any cell type in the body. Notably, any of these cell sources could be used in autologous cell therapy because they use of the patient's own cells, thereby avoiding the need for immunosuppression and the danger of disease transmission.

Scaffolds. Culturing cells in 3D scaffolds offers an in vitro alternative to the native extracellular matrix. A scaffold forms a structural and "cell-instructive" template for cells and the forming tissue. Scaffold materials, which are generally biodegradable and custom-designed to mimic the matrix of a specific tissue, develop 3D architectures suitable for cell seeding and cultivation. Material choices are guided by the need to restore cell-matrix interactions, direct cell alignment and migration and apply physical signals. Advanced scaffold designs are being developed to implement patterning, binding of ligands, sustained release of cytokines, and the structural and mechanical anisotropy intrinsic to specific tissues, such as heart muscle, or bone. An ideal scaffold would mimic the properties of a native tissue, have the anatomical shape and internal architecture of the graft being engineered, 7 interact dynamically with the cells, and become gradually replaced by newly synthesized native matrix.

Bioreactors. A bioreactor is much more than a Petri dish, where the cell environment is maintained by periodic changes of culture medium. In 3D cultures, media concentrations also change in space, because of diffusion gradients across the thickness of the tissue. In particular, oxygen penetration depth can be as little as $100 \mu \mathrm{m}$ for tissues such as heart ${ }^{10,11}$ or bone. ${ }^{7,8}$ Bioreactors are devices that control cell environment through enhanced mass transport to and from the cells, and they also control physical signals, such as hydrodynamic, mechanical, electrical changes. These changes, in turn, allow insights into cellular behavior through imaging and on line measurements. Ideally, a tissue engineering bioreactor should support cell viability and $3 \mathrm{D}$ organization by mechanisms similar to those in the native cell environment. ${ }^{8}$ In reality, bioreactors provide manipulation and control of the most important aspects of a given cell niche.

A few examples. Human ligaments were engineered by applying a combination of dynamic tension and torsion in a specialized bioreactor designed to mimic mechanical forces in the human knee. Interestingly, mechanical loading alone, without specific growth factors, induced cell alignment and accumulation of ligament-specific markers, in favor of alternate paths leading to cartilage or bone. ${ }^{12}$ Functional blood vessels were engineered by application of pulsatile fluid pressure designed to mimic that in native blood vessels: (i) hydrodynamic shear that acted on endothelial cells or smooth muscle cells like lumen or interstitial flow, (ii) cyclic pressure and (iii) circumferential and (iv) longitudinal stretching. ${ }^{13}$

As recently as 2008, an engineered airway made from allogeneic human trachea was implanted in a patient. ${ }^{14}$ The donor trachea was processed to remove cells and antigens and repopulated by the recipient's cells. The graft was then used to replace the whole left bronchus. The same year, another group removed the cells from rodent hearts by coronary perfusion with detergents. This biological template was then reseeded with cells and cultured in a bioreactor to obtain an engineered construct that generated minimal pumping function ( $2 \%$ of the adult heart) within one month of culture..$^{15}$ Most recently, two studies reported within the same week the successful engineering of a rodent lung by repopulating fully decellularized lungs. ${ }^{16,17}$ Remarkably, these engineered lungs functioned for several hours in vivo.

Anatomically shaped bone grafts were engineered using human mesenchymal stem cells and decellularized bone tissue matrix. The bone matrix, that was formed into the exact shape of a human jaw condyle using computer tomography images of a patient, was seeded with cells and cultured with interstitial flow in an "anatomical" bioreactor. By five weeks of cultivation, tissue growth was apparent from the formation of confluent layers of lamellar bone and osteoids and the assembly of mineralized bone matrix. For the first time within bone grafts of this size and complexity, cells were fully viable at a physiologic density.

\section{References}

1. Lysaght MJ and Reyes J. The Growth of Tissue Engineering, Tissue Eng 2001;7:485-93.

2. Langer R. and Vacanti J.P. Tissue engineering. Science 1993;260,920-6.

3. Cowin SC. How does Nature build a tissue? In Functional Tissue Eng: The role of Biomedchanics, F. Guilak, D. Butler, D. Mooney, S. Goldstein, eds. New York, Springer, 2003, pp 3-16.

4. Abbott A. Cell culture: Biology's new dimension, Nature 2003;424:870-2.

5. Kaplan D, Moon RT, and Vunjak-Novakovic G. It takes a village to grow a tissue. Nature Biotechnol 2005;23:1237-9. 
6. Zhang S. Beyond the Petri dish. Nature Biotechnol 2004;22:151-2.

7. Grayson WG, Fröhlich M, Yeager K, Bhumiratana S, Cannizzaro C, Wan LQ, Chan ME, Liu ME, Edward X, Guo EX, and VunjakNovakovic GV. Engineering anatomically shaped human bone grafts. Proc Nat Acad Sci USA 2009;107:3299-3304.

8. Burdick JA and Vunjak-Novakovic G. Engineered microenvironments for controlled stem cell differentiation. Originally published in Tissue Eng Part A 2009;15:205-219. Republished in a special edition of Adv Tissue Eng, featuring seminal work in tissue engineering (by invitation, in press)

9. Grayson W, Martens T, Eng G, Radisic M, and Vunjak-Novakovic G. Biomimetic approach to tissue engineering. Semin Cell Develop Biol 2009;20:665-73.

10. Radisic M, Park H, Chen F, Wang Y, Dennis R, Langer R, Freed LE and Vunjak-Novakovic G. Biomimetic approach to cardiac tissue engineering: Oxygen carriers and channeled scaffolds Tissue Eng 2006;12:2077-91. (Cover article, Editors choice in the Drug Discovery April 2007.)

11. Radisic M, Park H, Shing H, Consi T, Schoen F, Langer R, Freed LE, Vunjak-Novakovic G. Functional assembly of engineered myocardium by electrical stimulation of cardiac myocytes cultured on scaffolds Proc Nat Acad Sci 2004;101:18129-34. (Cover article)
12. Altman G, Horan RL, Martin I, Farhadi J, Stark, PRH, Volloch V, Richmond JC, Vunjak-Novakovic G, Kaplan DL. Cell Differentiation by mechanical stress. FASEB $J$ 2002;16: 270-2.

13. Niklason LE, Gao J, Abbott WM, Hirschi KK, Houser S, Marini $\mathrm{R}$ and Langer $\mathrm{R}$. Functional arteries grown in vitro. Science 1999;284:489.

14. Macchiarini P, Jungebluth P, Go T, Asnaghi MA, Rees LE, Cogan TA, Dodson A, Martorell J, Bellini S, Parnigotto PP, Dickinson SC, Hollander AP, Mantero S, Conconi MT, Birchall MA. Clinical transplantation of a tissue-engineered airway. Lancet 2008;372:2023-30.

15. Ott HC, Matthiesen TS, Goh SK, Black LD, Kren SM, Netoff TI, Taylor DA. Perfusion-decellularized matrix: using nature's platform to engineer a bioartificial heart. Nature Med 2008;14:213-21.

16. Ott HC, Clippinger B, Conrad C, Schuetz C, Pomerantseva I, Ikonomou L, Kotton D, Vacanti JP. Regeneration and orthotopic transplantation of a bioartificial lung. Nature Med 2010;16:927-33.

17. Petersen TH, Calle EA, Zhao L, Lee EJ, Gui L, Raredon MB, Gavrilov K, Yi T, Zhuang ZW, Breuer C, Herzog E, Niklason LE. Tissue-engineered lungs for in vivo implantation. Science 2010;329:538-41. 